\title{
Präventionspreis Frühkindliche Karies
}

Bis Ende September nimmt die „Initiative für eine mundgesunde Zukunft in Deutschland“ praxisorientierte Konzepte und Projekte entgegen, die die frühkindliche Kariesprävention nachweislich verbessert haben. Eine unabhängige Jury vergibt 3 Preise mit einer Gesamtdotierung von $5000 €$. Die Konzepte und Projekte sollen bundesweit umsetzbar sein, Erziehungsberechtigten und Betreuern die Wichtigkeit früher Kariesprävention verdeutlichen sowie letztlich zu einem verbesserten Ernährungs- und Mundhygieneverhalten bei den Kindern führen. Ganz bewusst halten die Initiatoren die Ausschreibe-Bedingungen offen, um möglichst viele verschiedene, interdisziplinäre Ideen einbeziehen zu können. Neben der Auszeichnung werden die Preisträger durch Öffentlichkeitsarbeit für ihr Projekt unterstützt. Zur Teilnahme aufgerufen sind Fachleute aus den Bereichen Gesundheitswesen, Public Health, Politikwissenschaften, Erziehungswissenschaften, Kommunikation und Medienwissenschaften. Auch staatliche und kommunale Institutionen wie Gesundheitsämter sind herzlich eingeladen. Bewerbungen können eingereicht werden bei der: „Initiative für eine mundgesunde Zukunft in Deutschland" Accente Communication $\mathrm{GmbH}$, Aarstraße 67, 65195 Wiesbaden, Tel.: 0611/40 80 6-0, Fax: 0611/40 80 6-99, E-mail: jonas.gobert@accente.de. Initiatoren des Preises sowie Gründer der „Initiative für eine mundgesunde Zukunft in Deutschland“ sind die Bundeszahnärztekammer (BZÄK) und CP GABA.

Nach einer Pressemitteilung der

CP GABA GmbH, Hamburg

Internet: www.gaba-dent.de 